\title{
Narrative review of lung cancer treatment at the time of COVID-19 pandemia: pitfall and issues
}

\author{
Francesca Casaluce, Cesare Gridelli \\ A.O.R.N. San Giuseppe Moscati, Contrada Amoretta, Avellino, AV, Italy \\ Contributions: (I) Conception and design: All authors; (II) Administrative support: All authors; (III) Provision of study materials or patients: All \\ authors; (IV) Collection and assembly of data: All authors; (V) Data analysis and interpretation: All authors; (VI) Manuscript writing: All authors; (VII) \\ Final approval of manuscript: All authors. \\ Correspondence to: Cesare Gridelli. Division of Medical Oncology, A.O.R.N. San Giuseppe Moscati Hospital, Contrada Amoretta, 83100 Avellino, Italy. \\ Email: cgridelli@libero.it.
}

\begin{abstract}
The severe acute respiratory syndrome coronavirus-2 (SARS-CoV-2), responsible for coronavirus disease 2019 (COVID-19) pandemic, has caused disruption in cancer care and research, changing daily management approach of cancer patients, above all for those affected by lung cancer disease. Unfortunately, its length and severity beyond today is still uncertain. This emerged viral pandemic has produced severe illness to overwhelm healthcare infrastructure, with worse impact on public health system and on providers of essential community services, and needing to ration medical equipment and interventions. Several data from across the world highlighted the susceptibility of patients affected by tumors to high severe infection and mortality from COVID-19. Lung cancer patients emerged as "frail" subgroup, mainly attributable to their immunosuppression, co-existing medical conditions and underlying pulmonary compromise. So, the lung cancer care was confounded by urgent need for intervention for most patients and the competing risk of life-threatening COVID-19 infection, and also influenced by competing needs for personnel, beds and equipment for urgent COVID-19 care. Clearly, no one model of care is possible during all pandemic phases or in all medical environments. In this review we will discuss on available data and their impact on prioritizing the individual management for lung cancer patients, with aim to protect them from COVID-19.
\end{abstract}

Keywords: Coronavirus disease 2019 (COVID-19); lung cancer, management.

Submitted May 08, 2020. Accepted for publication Sep 19, 2020.

doi: $10.21037 /$ tlcr-20-640

View this article at: http://dx.doi.org/10.21037/tlcr-20-640

\section{Introduction}

Identified in late 2019 as a cluster of pneumonia cases, the coronavirus disease 2019 (COVID-19) pandemic has no true precedent in modern times and is a rapidly evolving crisis worldwide, affecting more than 3 million people and forcing the medical community to reexamine every aspect of healthcare delivery (1). Given the systemic immunosuppressive state caused by malignancy and anticancer treatments, cancer patients face the double ordeals of disease and epidemic situation, appearing more likely vulnerable than general population. Up to date, data on clinical characteristics and outcomes of oncologic patients with COVID-19 infection are not detailed and still poor, representing a minority in all series reported. As suggested from early retrospective Chinese reports, cancer patients who are infected with COVID-19 are also associated to poor outcomes and higher occurrence of severe complications, and consequently higher mortality (2-5). About cancer care strategies, contrasting and limited data are available, but some series have shown higher risk of clinically severe events (HR: 4.079) for those patients who underwent treatment administration within 2 weeks (3), with chemotherapy as the worse ongoing treatment, followed by immunotherapy and at last by targeted therapy (5). Similarly, higher mortality and higher chances of having critical symptoms have reported 
after cancer surgery (6). A recent meta-analysis from 11 series revealed an overall pooled prevalence of COVID-19 cases in cancer patients of $2-3 \%$, more high than previously published results (7). Despite several limits—small series and selected population, above all-this value confirms cancer patients and cancer survivors as an important vulnerable population for the COVID-19 contamination. We present the following article in accordance with the Narrative Review reporting checklist (available at http://dx.doi.org/10.21037/ tlcr-20-640).

\section{The vulnerability of lung cancer patients}

Among all infected cancer patients, lung cancer patients result as the most representative subgroup, defining a special population during this outbreak, not only for their concomitant predisponing factors (older age, smoking habit, cardiopulmonary concomitant comorbidities, intensive therapies administered to treat their illness), but also because the clinical presentation of COVID-19 overlaps most of lung cancer symptoms, such as fever, cough, and dyspnea, making diagnosis of the virus infection very challenging. Furthermore, radiographic finding of COVID-19 can mirror like radiation pneumonitis and immunotherapy or targeted therapies induced pneumonitis. Interestingly, since the acute respiratory distress syndrome (ARDS) COVID-19 related seems to occur from an excess of cytokine production, this aberrant and noneffective host immune responses may resemble the ICI pneumonitis not only for clinical and radiological, but for pathophysiological features also (8). Anyway, the COVID-19 outbreak has imposed a rapid reorganization of cancer patients' management, with concomitant objectives of protect them from being infected by COVID-19 and from losing the chance to receive optimal, if not standard, cancer care. Generally, active prevention of infection (universal masking, social distancing, or isolation), timely conversion of treatment strategies, online and offline joint control, positive psychological counseling, early and rapid evaluation for symptoms suspicious for COVID-19, including testing for virus and chest computed tomography (CT) scan are some of important policies and actions from current management measures of COVID-19. On other hand, the limitation on system-based resource (including radiologic and surgery resources, with delay of those services), the patient safety issues, the staff exposure and the travel restrictions have inevitably occurred, with worse impact on public health system and on providers of essential community services.

Managing lung patients in this pandemic, many procedures were adopted at different points, including hospital admission (e.g., pre-triage by phone contact exploring any symptoms of disease or previous contact with people potentially infected and medical triage at visit), patient management (promote patient education, exploit remote visits to reduce COVID-19 contagion, outpatient visits allowed in selected cases, such as therapy administration), whose have certainly an impact on treatment decision and attend more attention to patients selection. Immediate efforts to modify therapeutic strategies for lung cancer patients are critical to mitigating patient risk and a guideline for the optimal management of lung patients urgently needs to be provided, individualizing treatment recommendations based on the epidemic situation of the patient's location and in combination with the patient's own condition. Clearly, it is not easy to find a cohesive collection of relevant journal articles and rapidly changing guidelines about COVID-19 in relation to lung cancer patients, with several questions remain still unanswered. The shortness of follow-up, the high selection of patient population, the small series of thoracic patients, the retrospectively collection of data, limited the global interpretation on how this infection affects patients on active treatment and how they ought to be treated as well as on how long to safely delay scans, treatments, surgery without long-term mortality impact. On other hand, extrapolation of first reported Chinese data to other countries may be problematic for two main reasons: the different prevalence of cancers subtype, and different clinical practice in China compared with Europe or North America, where most standard treatments occur in outpatient settings.

Born in March 2020, the first large global registry called TERAVOLT (Thoracic cancERs international coVid 19 cOLlaboraTion) is currently collecting characteristics and outcomes of patients with thoracic malignancies and COVID-19 infection (9). Notably, the registry included thoracic cancer patients with the COVID-19 diagnosis across all its heterogeneously manifestations, ranging from cases confirmed by reverse transcription-polymerase chain reaction (RT-PCR) test or suspected for presence of exposure and symptoms (fever $>37.5$ degrees $\mathrm{C}$, decrease of oximeter saturation of at least $5 \%$, cough, diarrhea, otitis, dysgeusia, myalgia, arthralgia, conjunctivitis, and rhinorrhea), to asymptomatic confirmed by RT-PCR test or radiologically suspected cases (lung imaging features consistent with COVID-19 pneumonia). For the first 200 
patients included, the median age was 68 years, mainly being male and current/former smokers. The majority of patients had non-small cell lung cancer (NSCLC) (75.5\%), stage IV disease, comorbidities in nearly $85 \%$ (mostly reported in hypertension), and were on oncological treatment, representing perfectly the real patients of daily clinical activity. Preliminary data from these population revealed an unexpectedly high mortality (death rate: $34.6 \%$ ), with pneumonitis and ARDS as the most frequent complications, and deaths mainly attributed to COVID-19 infection and not to their cancer. Hospitalization rates accounted more than $75 \%$ of patients, but less than $10 \%$ were admitted in intensive care units (ICU), allowing mechanical ventilation in only 5 patients, probably for shortages or institutional policies (9). Despite several limitations of this report (the short follow-up, the selected population with the majority of patients on treatment, few surgical cases, and the potential for a reporting bias) and clearly awaiting more data to confirm this, no anticancer treatments-included immunotherapy—were associated with higher mortality, and patients on tyrosine-kinase inhibitors (TKIs) had less risk to long hospitalization than others. Anyway, the higher mortality of lung cancer patients with COVID-19 relative to general population and other cancers-probably related to comorbid condition such as pulmonary disease and inherent risk of thrombosis of lung cancer, mirroring the same vascular effects of COVID-19-need more cautions for physician, whose final choice have to consider the heterogeneity and complexity of several variables about each patient (the individual risk of infection, the status of cancer, comorbidities and age, details of the treatment) and local epidemiology (the extent of the epidemic, the local healthcare structure capacity), but still hearing that a delays in some treatments might be potentially detrimental to patients survival.

\section{Management and treatment of lung cancer patients: how prioritizing?}

Generally, anticancer treatments are recommended in all patients with good clinical conditions, but in clinical practice of few months ago therapies were frequently delivered also in those defined "borderline" patients. Considering the current request of a "less risk therapy" to offer and made in condition of completely safety for patients, the selection of patient has a key-role, with a tendency of giving the less aggressive therapy, or simply the best supportive care, to those "frail" patients. The choice of treatment is changing and discussing with patients, whose still asking for therapies potentially reducing the risk of any adverse events (AEs).

Another impact of pandemic on lung cancer management is observed in terms of tumor biopsy or re-biopsy. Currently, the treatment-mainly for advanced diseaseis based on biological characteristics of the disease, and the collection of tissues to perform all the biomarkers analyses is need, as well as the re-biopsy at time of therapy failure is often advised, particularly after target therapies. During this infection, the possibility of perform, and particularly to repeat tumor biopsy, is complex and more challenging.

Third, we have a dramatically delay in clinical research. For several trials the enrollment has been stopped and for those open the inclusion of patients was dramatically reduced, mainly for limitation of staff attending this work. Patients still in treatment inside clinical study are managed in agreement with regulatory agencies, with aim to keep three following pivotal principals as well as possible: assuring the safely of trial participants, maintaining compliance with good clinical practice, and minimizing risk to trial integrity and quality of data.

Currently, majority of scientific societies created guidelines and practical suggestion on their relative website, prioritizing patients according to the kind of treatment and the stage of disease, in order to avoid any gathering condition which could increase the risk of infection for patients. The European Society of Medical Oncology (ESMO) has recently published on its website proposals of treatment recommendations for lung cancer patients in the era of COVID-19 (Table 1), prioritizing and maintaining all regimens with a clear survival benefit.

\section{Local and locally advanced NSCLC disease}

For localized and operable stage I disease, the first pitfall is represented by cancer surgery, the standard choice before pandemic, while stereotaxic radiosurgery (SBRT) limited only to medically inoperable patients. Generally, surgical service provision depends on COVID-19 burden, so mitigation and containment have to be obtained, prioritizing patients and pathology, and processing preparing and planning respectively. As recently reported at American Association for Cancer Research (AACR) 2020 virtual meeting, COVID-19 patients with lung cancerhematological and metastatic cancers too-had the highest frequency of severe events after surgery, and consequently surgery appeared to contribute to a high risk of poor outcomes (ICU admission, severe/critical symptoms, 
Table 1 Practical practice for lung cancer medical treatment during COVID-19 pandemic: ESMO guidelines

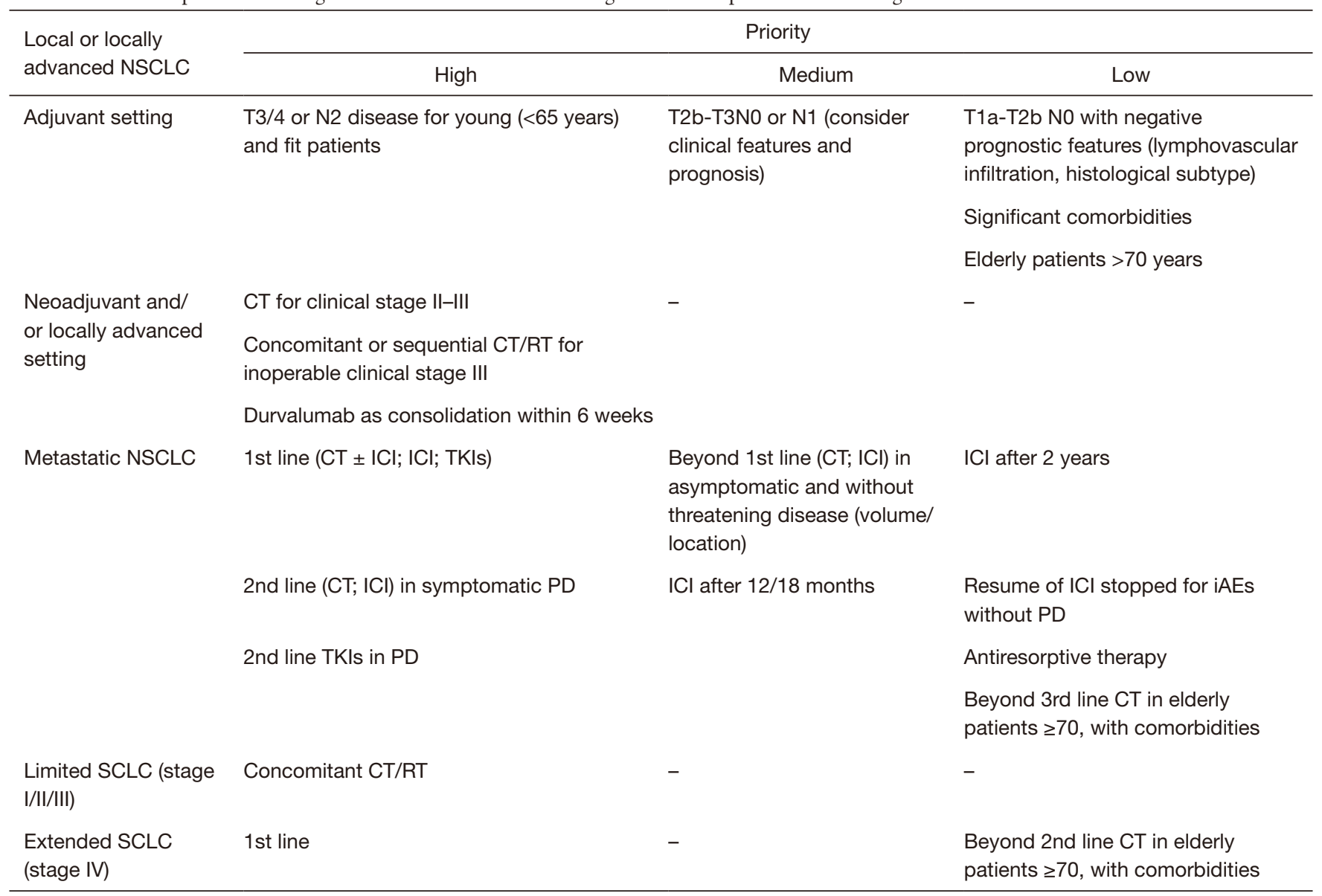

CT, chemotherapy; iAEs, immune related adverse events; ICI, immune-checkpoint inhibitors; NSCLC, non-small cell lung cancer; PD, progressive disease; RT, radiotherapy; SCLC, small cell lung cancer; TKIs, tyrosine kinase inhibitors.

invasive ventilation, death). On opposite, radiation therapy was not associated with increased risk of adverse outcomes (6). According to suggested detrimental effect of surgery and to average time-to-progression from early to advanced stages (10), several global society have reported guidelines for better prioritizing the management of thoracic cancer surgery at the time of COVID-19 outbreak, defining lung surgery as not elective choice-except for some urgent clinical cases (drainage \pm pleurodesis of pleural effusion, pericardial effusion, tamponade risk, evacuation of empyema-abscess) and diagnostic procedures-while promoting SBRT as the best option compared to a delayed surgery until operating rooms are available (11). Despite no level 1 equivalence data to surgery, the SBRT have unequivocal superiority over other non-operative options. If surgery resources are not available, the length of delay need to be estimated, hearing the successfulness of SBRT for small and/or peripheral lesions, while less promising approach for bulky central early lesions.

As adjuvant, treatments should be delivery and continue uninterrupted based on its curative-intent, but only after a comprehensive risks/benefits analysis, highly recommending its administration in N2 disease for young ( $<65$ years old) and fit patients (11). In a resource-limited setting or if patient preference, it may be reasonable to delay adjuvant chemotherapy for up to 4 months postoperatively, according to lack of differences in effectiveness and 5-year survivals of adjuvant chemotherapy respect to time of initiation after surgery, as reported in a retrospective analysis of more than 12,000 resected NSCLC patients (12).

Patients affected by locally advanced and resectable NSCLC (stage II-IIIA) represent a new issue to be alternatively managed in this emergency situation. Most of these patients who would normally undergo to thoracic 
surgery may instead receive non-surgical management, due to constrained resources (lack of ventilators for operating room) and risks associated with perioperative management. So, neoadjuvant/induction treatment should be started and/ or not stopped, enabling deferral of surgery by 3 months, preferring regimens with longer intervals between cycles and avoiding weekly infusions (11).

About caring for unresectable stage III NSCLC patients, radiotherapy concurrent or sequential to chemotherapy with curative intent should be reserved for those with adequate respiratory function, trying to minimize the exposure during the COVID-19 peak. The standard approach-consisting to concurrent chemoradiation, might increase chances of infections, including COVID-19, based on following factors: frequency and duration of treatments, healthcare providers, and above all the immunocompromising effects of concurrent chemoradiotherapy. So, the choice of administration modality should be carefully taken, considering that the established, but still modest, absolute survival benefit with concurrent might be outweighed by potential acute toxicities to be managed during the pandemic, and on other hand the better tolerated and reduced immunosuppressive effects of the sequential choice, paying the price of a longer total treatment time. Consolidation durvalumab should be started within 42 days after chemo-radiation, despite its routinely biweekly schedule (11).

\section{Systemic therapies for metastatic disease}

For advanced NSCLC, the impact on patients safety is influenced by what specifically treatment approaches they are ongoing, with different implications on the management of those being on chemotherapy from those on TKIs or immunotherapy. Anyway, the decision should be taken into consideration the line of therapy and their relationship between health risks and benefits, as well as whether to delay or suspend ongoing treatments for a period, miming the already known "drug holiday". According to ESMO guidelines, the first-line treatment, including chemotherapy alone or combined to immunotherapy, immunotherapy alone or TKIs should be always started or not stopped, with aim to improve prognosis, cancer-related symptoms and quality of life. At progression, a second line of TKI should be clearly started in oncogene-addicted diseases, while in all other cases the administration of chemo- or immuno-therapies as second line is strongly recommended only if patient is symptomatic, while the priority remain lower if asymptomatic and in absence of threatening disease (volume/location). The start of third and beyond lines of chemotherapy in patients at significant COVID-19-related risk (elderly or with comorbidities) is highly discouraged (11).

About chemotherapy, regimens with longer intervals and shorter duration should be preferred, and oral chemotherapy (etoposide, vinorelbine) should be considered for high risk patients (Eastern Cooperative Oncology Group Performance Status 2, elderly), with aim to limit hospital accesses. Pemetrexed as maintenance therapy might be stopped and shorted duration of chemotherapy (four cycles instead of six) should be discussed with patients. The prevention and management of neutropenic sepsis should be enforced, implementing home delivery of antibiotics and use of prophylactic granulocyte colony-stimulating factor if febrile neutropenia risk evaluated to be at least $10 \%$ or more. The offer of second lines of chemotherapy is obviously linked to patients' general conditions evaluation, and also to the first line regimen adopted. If patient was yet pretreated with immuno-agents and the only choice available consists in chemotherapy as docetaxel, the less toxic modified weekly schedule presents the critical problem of frequent hospitalizations.

About immunotherapy, the COVID-19 pandemic has raised several questions, with unfortunately few answers. First question-still the most frequent received from patients also-is whether the usage of immune drugs could influence the COVID-19 infection. Any evidence on the supposed protective effects of immunotherapy against COVID-19 infection is currently available. The opposite and hardest question to solve is about the more susceptibility of immuno-treated patients to COVID-19 infection and their higher risk to develop immune related AEs, with lung toxicities as the most relevant occurring in clinical practice. Since the acute respiratory stress syndrome COVID-19 related seems to occur from an excess of cytokine production, similarly to what happened during immuno-toxicities, one of main concern is related to the possible increasing risk of pneumonia in patients on treatment with immunotherapy, with consequently higher mortality. At present time, this hypothetical risk cannot be surely ruled out, lacking specifically data available, but having only few clinical reports who's not clarify if immunotherapy should be avoid during this pandemic. As recently reported from a retrospective Memorial Sloan Kettering Cancer Center experience, the PD-1 blockade exposure seems not be associated with increased risk of severity of COVID-19 infection in 69 patients with lung 
cancer (13).

In According to ESMO guideline, no data preclude the use of immunotherapy in lung cancer patients during COVID, confirming their recommended usage in first or subsequent lines, certainly with an accurate patient selection and with more attention to pneumonitis at CT scan even without fever (radiological features characterized by groundglass opacities, mimicking COVID-19 pneumonitis) and who need to differential diagnosis, performing COVID test (nasal swab) before start steroids. Similarly to chemotherapy, the adaptation of administration is highly encouraged to reduce hospital access, modifying or even delaying the cycles. Scheduled cycles with longer interval should be preferred (e.g., nivolumab $480 \mathrm{mg}$ every 4 weeks or pembrolizumab $400 \mathrm{mg}$ every 6 weeks), where allowed from National Regulatory Agency and when appropriate. The enlargement of interval (delay of next cycle, omit some scheduled cycle) is particularly encouraged in those on treatment from more than one year, as well as the definitive discontinuation for those treated at least for two years should be considered, after discussion with patient and clearly without evidence of relapse, despite lacking of prospective evidence (11). Finally, for patients on immunotherapy whose having temporary stopped for toxicity, re-introduction might be delayed in absence of disease progression.

About oncogenic-addicted disease, outcomes are strongly influenced by the continuous administration of targeted TKIs and their withdrawal might be detrimental, whose choice becoming even more relevant if taken for an asymptomatic pulmonary infection by COVID-19. So, the prosecution and/or the start of those oral drugs during the outbreak is highly recommended, but special attention should still be paid to the lung adverse events of some targeted drugs. Having many approved and available targeted drugs for the same oncogenic driver, the most appropriated drug to prefer in this pandemic should have better systemic and brain efficacy, but with less toxicities and consequently less hospital visits or seeing doctors. The selection regard to mitigate the risk of certain types of toxicities, above all some of lung toxicities [interstitial lung disease (ILD), pneumonia] showing radiological features characterized by ground-glass opacities, mimicking COVID-19 radiological characteristics and who need to differential diagnosis. About Epidermal Growth Factor Receptor (EGFR) TKIs, the third-generation osimertinib is the best choice to do, with superior efficacy and less toxicities than first-generation drugs (14). Comparing firstand second- generation EGFR-TKIs in terms of safety profile, withdrawal AEs and their consequently impact on discontinuation/reduction of drug doses-skin toxicities as the most common, followed by ILD, hepatotoxicity diarrhea and spleen cist-were significantly more frequent in afatinib/dacomitinib group compared to erlotinib/ gefitinib. The frequency of severe ILD was low (0.6-2.2\%) with all three EGFR-TKIs and did not differ significantly among them (15). About ALK inhibitors and severe AEs reported in clinical trials, alectinib was associated with lower rates, ranging from $29 \%$ to $45 \%$ as reported in ALEX and ALUR trials $(16,17)$, and promising itself as the ALK inhibitor to prefer in this pandemic.

In clinical practice, patients on targeted therapies generally should be isolated at home, get the targeted drugs one months per time adopting a drug delivery system for the prescription, and visit doctor every 2-3 months. When progression occurred, usage of second-third generation of TKIs for ALK rearranged disease is recommended, while the type of disease progression should be guide physician choice in management of EGFR-mutated disease. Particularly, if progression is gradual and/or local, the prosecution of TKI delaying of 1-2 months to see doctors should be the right choice, while if progression occur rapidly, COVID-19 infection should be excluded and liquid biopsy performed, prescribing osimertinib if positive and if not receiving itself as upfront choice, while switch to oral chemotherapy if negative.

An interesting question to debate regard patients with oncogenic-driver mutated lung cancer in treatment with a TKI and infected with COVID-19. As logically expected, treatment should not be reinitiated until symptoms of COVID-19 have resolved. Nowadays, few case reports are published, consisting in one EGFR-mutated and two ALKrearranged patients who maintained TKIs in the presence of COVID-19 interstitial pneumonia $(18,19)$. Notably, all these patients had a slight discomfort during the infection which not required intensive care. However, some points are to keep in mind. First, tumors harboring driver mutations usually affect young and never-smoker patients who represent a minority of cases we might face in clinical practice, consisting in older people with a smoking habit, affected by lung cancer without targetable mutations and candidate to other type of systemic treatments (chemotherapy, immunotherapy, or combination strategies), defining clearly as "frail" patients. Second, the interstitial pneumonia is a rare albeit potentially severe adverse events in patients receiving ALK-TKIs, a differential diagnosis between COVID-19 manifestation and a TKI-induced side effect must be taken into account. 
Since the radiological patterns of COVID-19 are evocative but not always diriment, a multidisciplinary discussion with radiologists is advisable in this subgroup of patients, and CT scan findings must be necessarily correlated with clinical and laboratory features.

\section{Conclusions}

As the evidence continues to rise, many questions remain unanswered: which lung patients get tested for COVID-19 and how often? What should be preferred between immunotherapy, chemotherapy, or chemo-immunotherapy? First, recommendation with respect to COVID-19 testing in asymptomatic lung cancer patients are currently not globally standardized. In Europe, patients with family members or caregivers who tested positive for COVID-19 should be tested before or during any cancer treatment, and if results positive and asymptomatic, a delay of 4 weeks should be considered before (re)starting the treatment, performing two negative tests at 1 -week interval. Due to their higher risk for severe COVID-19 infection for concomitant other factors (age, co-morbidities, structural lung disease, likely prior smoking history, as well as their cancer and its treatment) baseline COVID-19 testing for all patients affected by lung cancer should be recommended, as well as considering bronchoscopy to increase testing sensitivity in those with negative RT-PCR and a concerning CT chest or symptoms (20).

Second, the fight of chemo $v s$. immuno as the best and less toxic treatment choice is still open, with no evidence that myelosuppression of chemotherapy could increase the risk of COVID infection, as well as no clear role of immunotherapy and its effect as protective or detrimental.

So, existing data - with high levels of bias-do not yet answer all these questions. On other hand, data from such small, highly selected, and often flawed case series could have unintended consequences by unduly influencing physicians' practice or clinical guidelines, particularly if conclusions are cited and propagated without either context or the acknowledgement of those high levels of uncertainty (21).

\section{Acknowledgments}

Funding: None.

\section{Footnote}

Reporting Checklist: The authors have completed the
Narrative Review reporting checklist. Available at http:// dx.doi.org/10.21037/tlcr-20-640

Conflicts of Interest: Both authors have completed the ICMJE uniform disclosure form (available at http://dx.doi. org/10.21037/tlcr-20-640). CG reports as speaker bureau and advisory board member from Astra Zeneca, BMS, MSD, Roche, Menarini, Novartis. The authors have no other conflicts of interest to declare.

Ethical Statement: The authors are accountable for all aspects of the work in ensuring that questions related to the accuracy or integrity of any part of the work are appropriately investigated and resolved.

Open Access Statement: This is an Open Access article distributed in accordance with the Creative Commons Attribution-NonCommercial-NoDerivs 4.0 International License (CC BY-NC-ND 4.0), which permits the noncommercial replication and distribution of the article with the strict proviso that no changes or edits are made and the original work is properly cited (including links to both the formal publication through the relevant DOI and the license). See: https://creativecommons.org/licenses/by-nc-nd/4.0/.

\section{References}

1. WHO Director-General's opening remarks at the media briefing on COVID-19 - 11 March 2020. World Health Organization. Available online: https://www.who.int/dg/ speeches/detail/who-director-general-s-opening-remarksat-the-media-briefing-on-covid-19---11-march-2020

2. Guan WJ, Ni ZY, Hu Y, et al. Clinical characteristics of coronavirus disease 2019 in China. N Engl J Med 2020;382:1708-20.

3. Liang W, Guan W, Chen R, et al. Cancer patients in SARS-CoV-2 infection: a nationwide analysis in China. Lancet Oncol 2020;21:335-7.

4. Yu J, Ouyang W, Chua MLK, et al. SARS-CoV-2 transmission in patients with cancer at a tertiary care hospital in Wuhan, China. JAMA Oncol 2020;6:1108-10.

5. Zhang L, Zhu F, Xie L, et al. Clinical characteristics of COVID-19 infected cancer patients; A retrospective case study in three hospitals within Wuhan, China. Ann Oncol 2020;31:894-901.

6. Dai M, Liu D, Liu M, et al. Patients with cancer appear more vulnerable to SARS-COV-2: A multi-center Study during the COVID-19 outbreak. Cancer Discov. 
2020;10:783-91.

7. Desai A, Sachdeva S, Parekh T, et al. COVID-19 and Cancer: Lessons From a Pooled Meta-Analysis. JCO Glob Oncol 2020;6:557-9.

8. Addeo A, Obeid M, Friedlaender A. COVID-19 and lung cancer: risks, mechanisms and treatment interactions. J Immunother Cancer 2020;8:e000892.

9. Garassino MC, Whisenant JG, Huang LC, et al. COVID-19 in patients with thoracic malignances (TERAVOLT): first results of an international, registrybased cohort study. Lancet Oncol 2020;21:914-22.

10. Yuan P, Cao JL, Rustam A, et al. Time-to-Progression of NSCLC from Early to Advanced Stages: An Analysis of data from SEER Registry and a Single Institute. Sci Rep 2016;6:28477.

11. Banna G, Curioni-Fontecedro A, Friedlaender A, et al. How we treat patients with lung cancer during the SARS$\mathrm{CoV}-2$ pandemic: primum non nocere. ESMO Open 2020;5:e000765.

12. Salazar MC, Rosen JE, Wang Z, et al. Association of Delayed Adjuvant Chemotherapy With Survival After Lung Cancer Surgery. JAMA Oncol 2017;3:610-9.

13. Luo J, Rizvi H, Egger JV, et al. Impact of PD-1 blockade on severity of COVID-19 in patients with lung cancers. Cancer Discov 2020;10:1121-8.

14. Soria JC, Ohe Y, Vansteenkiste J, et al. Osimertinib in

Cite this article as: Casaluce F, Gridelli C. Narrative review of lung cancer treatment at the time of COVID-19 pandemia: pitfall and issues. Transl Lung Cancer Res 2021;10(1):475-482. doi: $10.21037 /$ tlcr-20-640
Untreated EGFR-Mutated Advanced Non-Small-Cell Lung Cancer. N Engl J Med 2018;378:113-25.

15. Takeda M, Okamoto I, Nakagawa K. Pooled safety analysis of EGFR-TKI treatment for EGFR mutation-positive non-small cell lung cancer. Lung Cancer 2015;88:74-9.

16. Peters S, Camidge DR, Shaw AT, et al. Alectinib versus Crizotinib in Untreated ALK-Positive Non-Small-Cell Lung Cancer. N Engl J Med 2017;377:829-38.

17. Novello S, Mazières J, Oh IJ, et al. Alectinib versus chemotherapy in crizotinib-pretreated anaplastic lymphoma kinase (ALK)-positive non-small-cell lung cancer: results from the phase III ALUR study. Ann Oncol 2018;29:1409-16.

18. Zhang H, Huang Y, Xie C. The Treatment and outcome of a lung cancer patient infected with SARS-CoV-2.J Thorac Oncol 2020;15:e63-4.

19. Leonetti A, Facchinetti F, Zinelli T, et al. COVID-19 in lung cancer patients receiving ALK/ROS1 inhibitors. Eur J Cancer 2020;132:122-4.

20. Passaro A, Peters S, Mok TSK, et al. Testing for COVID-19 in lung cancer patients. Ann Oncol 2020;31:832-4.

21. Robinson AG, Gyawali B, Evans G. COVID-19 and cancer: do we really know what we think we know? Nat Rev Clin Oncol 2020,17:386-8. 